\title{
NONLINEAR RESPONSE OF COMPOSITE PANELS UNDER COMBINED ACOUSTIC EXCITATION AND AERODYNAMIC PRESSURE
}

\author{
K. Abdel-Motagaly, B. Duan, and C. Mei \\ Department of Aerospace Engineering, \\ Old Dominion University, Norfolk, VA 23529-0247
}

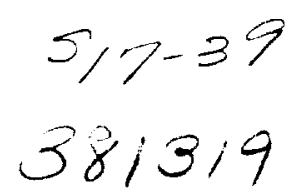

\begin{abstract}
A finite element formulation is presented for the analysis of large deflection response of composite panels subjected to aerodynamic pressure at supersonic flow and high acoustic excitation. The first-order shear deformation theory is considered for laminated composite plates, and the von Karman nonlinear straindisplacement relations are employed for the analysis of large deflection panel response. The first-order piston theory aerodynamics and the simulated Gaussian white noise are employed for the aerodynamic and acoustic loads, respectively. The nonlinear equations of motion for an arbitrarily laminated composite panel subjected to a combined aerodynamic and acoustic pressures are formulated first in structure node degrees-of-freedom. The system equations are then transformed and reduced to a set of coupled nonlinear equations in modal coordinates. Modal participation is defined and the in-vacuo modes to be retained in the analysis are based on the modal participation values. Numerical results include root mean square values of maximum deflections, deflection and strain response time histories, probability distributions, and power spectrum densities. Results showed that combined acoustic and aerodynamic loads have to be considered for panel analysis and design at high dynamic pressure values.
\end{abstract}

\section{INTRODUCTION}

Aircraft and spacecraft skin-panels are subjected simultaneously to high levels acoustic (sonic fatigue) and aerodynamic (panel flutter) pressures. ${ }^{1.2}$ Sonic fatigue and panel flutter have been the major design considerations for aircraft, spacecraft and missiles since the late nineteen sixties. An excellent review of sonic fatigue technology up to 1989 was given by Clarkson. ${ }^{3}$ Various types of pressure loads, developments of theoretical methods, and comparisons of experimental results with theories and nomographs were given. Recently, Wolfe et al. ${ }^{4}$ gave reviews in-depth of sonic fatigue design guides, classical and finite element approaches, and identification technology. Experimental investigation of nonlinear beams and plates, and multimodal fatigue model were also reported. Sonic fatigue design guides have been developed by Rudder and Plumblee ${ }^{5}$ for isotropic metallic and by Holehouse $^{6}$ for graphite-epoxy composite aircraft structures. The design guides, however, were based on the semiempirical test data or the simplified single-mode approach. Vaicaitis et al. have developed a Galerkin-like procedure (PDE and modal method) and a time domain Monte Carlo approach for the nonlinear response of isotropic $^{1.2}$ and composite ${ }^{7,8}$ panels to acoustic and thermal loads.

An excellent survey of nonlinear panel flutter through 1970 was given by Dowell. ${ }^{9}$ The vast amount of theoretical literature on panel flutter were grouped into four categories based on the linear or nonlinear structure theories, and the two aerodynamic theories (quasi-steady first-order piston or full linearized inviscid potential flow). The partial differential equations (PDE), the Galerkin's method and the numerical simulation results showed that a minimum of six modes are needed for a converged limit cycle amplitude response. Bismarck-Nasr ${ }^{10}$ gave a review of the linear panel flutter using the finite element methods. Recently, a review of various analytical methods and experimental results of nonlinear panel flutter at supersonic and hypersonic speeds was given by Mei et al. ${ }^{11} \mathrm{An}$ approach for the design of flutter-free surface panels using the quasi-static Ackeret aerodynamic theory was documented by Laurenson and McPherson. ${ }^{12}$ A exhaustive search of the literature reveals that there is no study of nonlinear panel response to combined acoustic and aerodynamic loads.

When a flight vehicle travels at supersonic speeds, panel flutter caused by aerodynamic pressure is not the only form of dynamic instability. The surface panels also experience high frequency random pressure fluctuations (sonic fatigue). ${ }^{1.2}$ This paper presents a finite element formulation for the analysis of nonlinear large deflection response of composite panels subjected to high acoustic excitation and aerodynamic pressure at supersonic speeds. The firstorder shear deformation theory is considered for the laminated composite plates. The von Karman nonlinear strain- 
displacement relations are employed for the large deflection response of the panel. Simulated Gaussian white noise and the first order piston theory aerodynamics are employed for the acoustic and aerodynamic loads. The nonlinear equations of motion for an arbitrarily laminated composite panel subjected to a combined high acoustic and aerodynamic loads are formulated first in the structure node degrees-of-freedom (DOF). The system equations are then transformed and reduced to a set of coupled nonlinear equations in modal coordinates. Numerical integration is employed to obtain the panel response. Examples are given for an isotropic and a composite panel at various combinations of sound pressure level and dynamic pressure.

\subsection{Equations of Motion in Structure Node DOF}

\section{FORMULATION}

The inplane strain, curvature and shear strain vectors based on the von Karman large deflection and the first order shear deformation theories are given by

$$
\begin{aligned}
& \left\{\varepsilon^{o}\right\}=\left\{\begin{array}{c}
o \\
\varepsilon_{m}
\end{array}\right\}+\left\{\varepsilon_{b}^{o}\right\}=\left\{\begin{array}{c}
u, x \\
v_{, y} \\
u, y+v_{, x}
\end{array}\right\}+\left\{\begin{array}{c}
w_{, x}^{2} / 2 \\
w_{, y}^{2} / 2 \\
w_{, x}, y
\end{array}\right\} \\
& \{\kappa\}=\left\{\begin{array}{c}
\psi_{x, x} \\
\psi_{y, y} \\
\psi_{x, y}+\psi_{y, x}
\end{array}\right\}, \quad\{\gamma\}=\left\{\begin{array}{l}
w_{, y}+\psi_{y} \\
w_{, x}+\psi_{x}
\end{array}\right\}
\end{aligned}
$$

where $u, v$ and $w$ are the inplane and transverse displacements, respectively. $\Psi_{x}$ and $\Psi_{y}$ are the rotations of the normal to the midsurface about the $y$ - and $x$-axes, respectively. The subscripts $m$ and $b$ denote membrane (inplane) and bending components, respectively. The constitutive equations for a laminated composite plate are

$$
\left.\left\{\begin{array}{l}
N \\
M
\end{array}\right\}=\left[\begin{array}{ll}
A & B \\
B & D
\end{array}\right]\left\{\begin{array}{c}
\varepsilon^{o} \\
\kappa
\end{array}\right\}, \quad\{Q\}=\left[A_{s}\right\} \gamma\right\}
$$

where $[A],[B],[D]$ and $\left[A_{s}\right]$ are the stretching, bending-stretching coupling, bending, and shear stiffnesses, respectively. The quasi-steady first-order piston aerodynamic theory is employed for the aerodynamic pressure at high supersonic Mach number $\left(M_{\infty}>1.6\right)$. The aerodynamic pressure is given by ${ }^{9}$

$$
\Delta p=-\frac{2 q_{a}}{\beta}\left(w_{, x}+\frac{M_{\infty}^{2}-2}{M_{\infty}^{2}-1} \frac{1}{V_{\infty}} w_{, t}\right)
$$

where $\mathrm{q}_{\mathrm{a}}=\rho_{\mathrm{a}} \mathrm{V}_{\infty}{ }^{2} / 2$ is the free stream dynamic pressure, $\rho_{\mathrm{a}}$ the air density, $\mathrm{V}_{\infty}$ the velocity and $\beta=\sqrt{M_{\infty}^{2}-1}$.

Using the Hamilton's principle and the finite element expressions, the system equations of motion for a composite plate subjected to aerodynamic pressure and high acoustic excitation can be expressed as ${ }^{13,14}$

$$
\frac{1}{\omega_{o}^{2}}[M]_{b}\left\{\ddot{W_{b}}\right\}+\frac{g_{a}}{\omega_{o}}[G]\left\{\dot{W}_{b}\right\}+\left(\left[K_{L}\right]+\left[K_{N L}\right]\right)\left\{W_{b}\right\}=\left\{P_{b}(t)\right\}
$$

where $\omega_{o}=\left(D_{110} / \rho h a^{4}\right)^{1 / 2}$ is a reference frequency and $\rho, \mathrm{h}$ and a are the panel density, thickness and length, respectively. The value $D_{110}$ is the first entry in laminate bending rigidity $[D]$ calculated when all of the fibers of the composite layers are aligned in the airflow $x$-direction. The $[M]_{b},[G]$ and $\left\{P_{b}\right\}$ are the system mass matrix, aerodynamic damping matrix and load vector due to random acoustic pressure, respectively. The linear and nonlinear system stiffness matrices are given by

$$
\begin{gathered}
{\left[K_{L}\right]=\lambda\left[A_{a}\right]+[K]_{b}+\left[K_{S}\right]-\left[K_{B} \mathrm{IK}_{m}^{-1}\left[K_{B}\right]^{T}\right.} \\
{\left[K_{N L}\right]=-\left[K_{B} \mathrm{IK}_{m}^{-1}[K 1]_{m b}+\left[K 1_{B}\right]+\left[K 1_{N m}\right]+\left[K 1_{N b}\right]+[K 2]-[K 1]_{b m}[K]_{m}^{-1}\left(\left[K_{B}\right]^{T}+[K 1]_{m b}\right)\right.}
\end{gathered}
$$

where the nondimensional dynamic pressure and nondimensional aerodynamic damping are given by 


$$
\lambda=\frac{2 q_{a} a^{3}}{\beta D_{110}}, g_{a}=\sqrt{\lambda C_{a}}
$$

where $C_{a}=\mu\left(M_{\infty}^{2}-2\right)^{2} / \beta\left(M_{\infty}^{2}-1\right)^{2}$ is the aerodynamic coefficient and $\mu=\rho_{a} a / \rho h$ is the mass ratio. For high supersonic speeds $\mathrm{M}_{\infty} \gg 1$, Dowell ${ }^{9}$ approximated $C_{a} \approx \mu / M_{\infty} .\left[\mathrm{A}_{\mathrm{a}}\right]$ is the system aerodynamic influence matrix, and $[\mathrm{K} 1]$ and $[\mathrm{K} 2]$ are the first-order and second-order nonlinear stiffness matrices which depend linearly and quadratically upon the unknown system displacement vector $\{\mathrm{W}\}=\left\{\left\{\mathrm{W}_{\mathrm{b}}\right\},\left\{\mathrm{W}_{\mathrm{m}}\right\}\right\}^{\mathrm{T}}$. The subscripts $\mathrm{B}, \mathrm{N}_{\mathrm{m}}$ and $\mathrm{N}_{\mathrm{b}}$ denote that the corresponding stiffness matrix is due to the laminate extension-bending stiffness $[B]$, membrane force components $\left\{N_{m}\right\}=[A]\left\{\varepsilon_{m}{ }^{\circ}\right\}$ and $\left\{N_{b}\right\}=[B]\{\kappa\}$, respectively, and the subscripts a and s denote aerodynamic and shear deformation, respectively. The derivation of Eq. (4) can be referred to references 13 and 14 . In the absence of acoustic loading, $\left\{\mathrm{P}_{\mathrm{b}}(\mathrm{t})\right\}=0$, Eq. (4) reduces to nonlinear panel flutter at supersonic speeds; On the other hand, by setting $\lambda=g_{a}=0$, Eq. (4) describes nonlinear random response of a composite panel subjected to high acoustic excitations. According to the authors' knowledge, this is the first attempt in investigating of nonlinear response of panels subjected to a combined acoustic and aerodynamic pressures.

For a given set of $\lambda$ and $C_{a}$ (or $\mu M_{\infty}$ ), Eq. (4) can be solved by numerical integration in the structure node DOF for combined load case. This approach has been carried out for sonic fatigue analysis with simulated random loads. ${ }^{15,16}$ It turned out to be computationally costly due to: (i) at each time step, the element nonlinear stiffness matrices are evaluated and the system nonlinear stiffness matrix $\left[\mathrm{K}_{\mathrm{NL}}\right]$ is assembled and updated, (ii) the number of structure node DOF of $\left\{\mathrm{W}_{\mathrm{b}}\right\}$ is usually very large, and (iii) the time step of integration should be extremely small.

An efficient solution procedure is to transform Eq. (4) into the modal coordinates with a modal reduction. This approach is presented as follows.

\subsection{Equations of Motion in Modal Coordinates}

Express the panel deflection as a linear combination of some known base functions as

$$
\left\{W_{b}\right\}=\sum_{r=1}^{n} q_{r}(t)\left\{\phi_{r}\right\}=[\Phi\}\{q\}
$$

where the number of retained linear in-vacuo modes, $n$, is much smaller than the number of structure node DOF in bending, $\left\{W_{\mathrm{b}}\right\}$. The normal mode $\left\{\phi_{\mathrm{r}}\right\}$, which is normalized with the maximum component to unity, and the linear natural frequency $\omega_{\text {, }}$ are obtained from the linear vibration of the system

$$
\frac{\omega_{r}^{2}}{\omega_{o}^{2}}[M]_{b}\left\{\phi_{r}\right\}=\left([K]_{b}+\left[K_{s}\right]-\left[K_{B} \mathbf{I}\right]_{m}^{-1}\left[K_{B}\right]^{T}\right)\left\{\phi_{r}\right\}
$$

A small number of most contributing modes to be retained in the analysis can be determined from the modal participation value which is defined as

$$
\text { Participation of the } \mathrm{r}^{\text {th }} \text { mode }=\operatorname{RMS}\left(\mathrm{q}_{\mathrm{r}}\right) / \sum_{s=1}^{n} \mathrm{RMS}\left(\mathrm{q}_{\mathrm{s}}\right)
$$

Since matrices $[\mathrm{K} 1]_{\mathrm{mb}},\left[\mathrm{K} 1_{\mathrm{B}}\right],\left[\mathrm{K} 1_{\mathrm{Nb}}\right]$ and $[\mathrm{K} 2]$ are all functions of the unknown bending DOF $\left\{\mathrm{W}_{\mathrm{b}}\right\}$, they can now be expressed as the sum of products of modal coordinates and nonlinear modal stiffness matrices as

$$
\left([K 1]_{m b},\left[K 1_{B}\right],\left[K 1_{N b}\right],[K 2]\right)=\sum_{r=1}^{n} q_{r}\left([K 1]_{m b}^{(r)},\left[K 1_{B}\right]^{(r)},\left[K 1_{N b}\right]^{(r)}, \sum_{s=1}^{n} q_{s}[K 2]^{(r s)}\right)
$$

where the super-indices of those nonlinear modal stiffness matrices denote that they are assembled from the corresponding element nonlinear stiffness matrices. Those element nonlinear stiffness matrices are evaluated with the corresponding element components $\left\{w_{b}\right\}^{(r)}$ obtained from the known system linear mode $\left\{\phi_{r}\right\}$. Therefore, the nonlinear modal stiffness matrices are constant matrices. The matrix $\left[\mathrm{K} 1_{\mathrm{Nm}}\right]$, however, is a linear function of the inplane DOF $\left\{W_{m}\right\}$ which consists of two terms as 


$$
\begin{aligned}
\left\{W_{m}\right\} & =-[K]_{m}^{-1}\left(\left[K_{B}\right]^{T}+[K 1]_{m b}\right)\left\{W_{b}\right\} \\
& =-[K]_{m}^{-1}\left(\left[K_{B}\right]^{T}+\sum_{s=1}^{n} q_{s}[K 1]_{m b}^{(s)}\right) \sum_{r=1}^{n} q_{r}\left\{\phi_{r}\right\} \\
& =-\sum_{r=1}^{n} q_{r}\left\{\phi_{r}\right\}_{m}-\sum_{r=1}^{n} \sum_{s=1}^{n} q_{r} q_{s}\left\{\phi_{r s}\right\}_{m}
\end{aligned}
$$

where the two inplane modes corresponding to the $\mathrm{r}^{\text {th }}$ bending mode $\left\{\phi_{\mathrm{r}}\right\}$ are given by

$$
\left\{\phi_{r}\right\}_{m}=[K]_{m}^{-1}\left[K_{B}\right]\left\{\phi_{r}\right\}, \quad\left\{\phi_{r s}\right\}_{m}=[K]_{m}^{-1}[K 1]_{m b}^{(r)}\left\{\phi_{s}\right\}
$$

The nonlinear stiffness matrix $\left[\mathrm{K} 1_{\mathrm{Nm}}\right]$ can be expressed as the sum of two nonlinear modal stiffness matrices as

$$
\left[K 1_{N m}\right]=-\sum_{r=1}^{n} q_{r}\left[K 1_{N m}\right]^{(r)}-\sum_{r=1}^{n} \sum_{s=1}^{n} q_{r} q_{s}\left[K 2_{N m}\right]^{(r s)}
$$

The nonlinear modal stiffness matrices $\left[\mathrm{K} 1_{\mathrm{Nm}}\right]^{(\mathrm{s})}$ and $\left[\mathrm{K} 2_{\mathrm{Nm}}\right]^{(\mathrm{rs})}$ are constant matrices and they are assembled and evaluated with the known inplane modes $\left\{\phi_{\mathrm{r}}\right\}_{\mathrm{m}}$ and $\left\{\phi_{\mathrm{rs}}\right\}_{\mathrm{m}}$, respectively. Equation (4) is thus transformed to the reduced modal coordinates as

$$
\frac{1}{\omega_{o}^{2}}[\bar{M}]\{\ddot{q}\}+\frac{g_{a}}{\omega_{o}}[\bar{G}]\{\dot{q}\}+2 \xi, \omega, \frac{\overline{M_{r}}}{\omega_{o}^{2}}[I]\{\dot{q}\}+\left(\left[K_{L}\right]+\left[K_{q}\right]+\left[K_{q q}\right] \mathfrak{h q}\right\}=\{\bar{P}(t)\}
$$

where the modal matrices are given by

and the quadratic and cubic terms are

$$
\left([M],[G],\left[K_{L}\right]\right)=[\Phi]^{T}\left([M]_{b},[G],\left[K_{L}\right]\right)[\Phi]
$$

$$
\begin{aligned}
& \left.\left.\left[K_{q}\right]\{q\}=[\Phi]^{T} \sum_{r=1}^{n} q_{r}\left(-\left[K{ }_{B} \mathbf{I}\right]_{m}^{-1}[K]_{m b}^{(r)}+\left[K 1_{B}\right]^{(r)}-\left[K 1_{N m}\right]^{(r)}+\left[K 1_{N b}\right]^{(r)}-[K 1]_{b m}^{(r)}[K]_{m}^{-1}[K B]\right]^{T}\right) \Phi\right]\{q\} \\
& \left.\left[K_{q q}\right]\{q\}=[\Phi]^{T} \sum_{r=1}^{n} \sum_{s=1}^{n} q_{r} q_{s}\left([K 2]^{(r s)}-\left[K 2_{N m}\right]^{(r s)}-[K 1]_{b m}^{(r)}[K]_{m}^{-1}[K 1]_{m b}^{(s)}\right)\right)^{(T)}\{q\}
\end{aligned}
$$

and the modal force is

$$
\{P(t)\}=[\Phi]^{T}\left\{P_{b}(t)\right\}
$$

A structural modal damping $2 \xi_{r} \omega_{r} \frac{\bar{M}_{r}}{\omega_{o}^{2}}[I]$ has been added to Eq. (14), and $\xi_{r}$ is the modal damping ratio which can be determined experimentally or from the data base of structures of similar construction. The nonlinear response for a given panel at certain dynamic pressure $\lambda$ and damping parameters $C_{a}$ and $\xi_{r}$ can be determined from Eq. (14) by any numerical integration scheme. The advantages in using Eq. (14) are: (i) there is no need to assemble and update the quadratic and cubic nonlinear terms since all the nonlinear modal matrices are constant matrices, and (ii) the number of modal equations, $n$, is small.

\subsection{Random Surface Pressure}

The input acoustic excitation is assumed to be band-limited Gaussian random noise and uniformly distributed over the structural surface. The power spectrum density (PSD) has the form

$$
\begin{array}{rlrl}
S(f) & =p_{o}^{2} 10^{S P L / 10} & 0 \leq f \leq f_{c} \\
& =0 & & \text { otherwise }
\end{array}
$$

where $p_{0}$ is the reference pressure, $p_{0}=2.9 \times 10^{-9} \mathrm{psi}\left(0.00002 \mathrm{~N} / \mathrm{m}^{2}\right)$, SPL is the sound pressure level in decibels and $f_{c}$ is the selected band width. The formulation presented in Eq. (14), however, is not limited to stationary Gaussian excitation. It can also handle nonstationary, non-Gaussian random loading which the high speed flight vehicles would probably experience. With recorded flight high frequency pressure 
fluctuations, random panel response can be determined much realistically by numerical integration of Eq. (14).

\section{EXAMPLES AND DISCUSSION}

The nonlinear system equations presented in Eq. (4) are general in the sense that they are applicable for rectangular ${ }^{13.15}$ or triangular ${ }^{14}$ finite elements. The finite element employed in the examples is the three-node triangular Mindlin (MIN3) plate element with improved transverse shear. ${ }^{17}$ The shear correction factor is defined as $\alpha_{s}=1 /\left(1+0.5 \sum_{i=4,9} k_{s i} / \sum_{i=4,9} k_{b i j}\right)$. The MIN3 element has a total of 15 DOF, 5 at each apex node. The bending node DOF

$\left\{w_{b}\right\}$ comprise of transverse displacements and normal rotations ( $w, \Psi_{x}$ and $\Psi_{y}$ ) and the inplane node DOF $\left\{w_{m}\right\}$ comprise of inplane displacements ( $u$ and $v$ ). Nonlinear response are obtained for a square isotropic plate and a rectangular composite plate. An aerodynamic coefficient $\mathrm{C}_{\mathrm{a}}=0.01$ and a modal damping ratio $\xi_{r}=0.01, \mathrm{r}=1$ to $\mathrm{n}$ are used in the examples.

\subsection{Square Isotropic Plate}

A simply supported square aluminum panel under the combined acoustic and aerodynamic pressures is studied in detail. The plate is of $12 \times 12 \times 0.040$ in. $(30.5 \times 30.5 \times 0.1 \mathrm{~cm})$ with immovable inplane edge conditions $u(0, y)=$ $u(a, y)=v(x, 0)=v(x, a)=0$. The material properties are $E=10 \mathrm{Msi}(68.89 \mathrm{GPa})$ and $v=0.3$. The plate is modeled with a $12 \times 12$ mesh or $288 \mathrm{MIN} 3$ elements. The number of structural node DOF $\left\{\mathrm{W}_{\mathrm{b}}\right\}$ is 407 for the system equations given in Eq. (4). It is well know that 6 modes in the airflow direction are needed for a converged limit cycle response for panel flutter and the lowest few symmetrical modes are needed for the uniform input random pressure distribution for sonic fatigue. Therefore the modes considered for the combined aerodynamic and acoustic loads are $(1,1)$ to $(6,1)$ for the panel flutter and $(1,1),(1,3),(3,1)$ and $(3,3)$ for the sonic fatigue analysis. No modal participation calculations are needed for this well studied problem in panel flutter and sonic fatigue. The root mean square (RMS) maximum deflections to plate thickness versus the nondimensional dynamic pressure $\lambda$ at SPL of 0 , 100,110 and $120 \mathrm{~dB}$ are shown in Fig. 1.

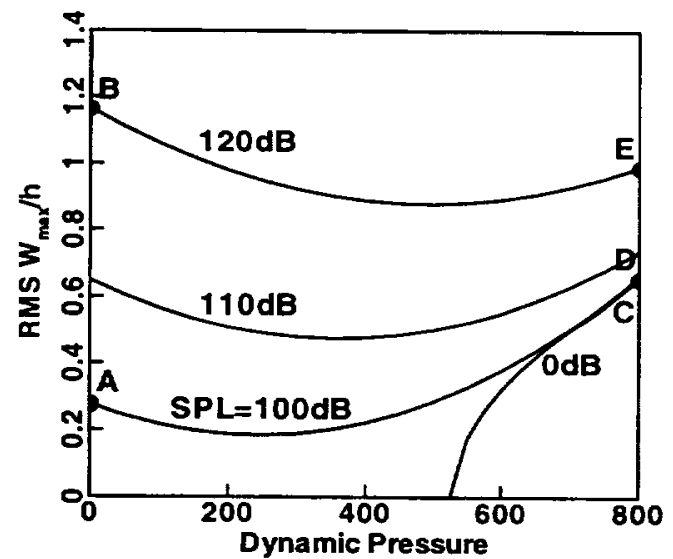

Fig. 1 RMS maximum deflection of a simply supported square isotropic plate

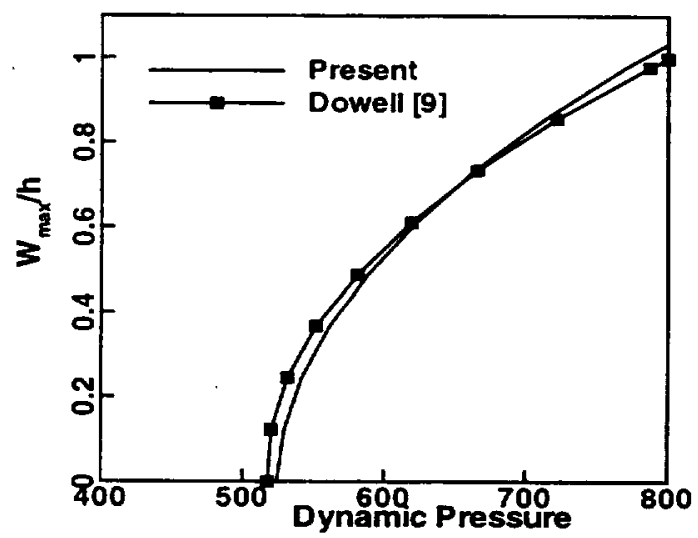

Fig. 2 Comparison of limit cycle amplitude for a simply supported square isotropic plate

The curve with the null acoustic pressure ( $0 \mathrm{~dB}$ SPL) is the conventional panel flutter limit cycle oscillations, and the RMS deflections at null dynamic pressure $(\lambda=0)$ are the conventional nonlinear panel response to acoustic excitations. The accuracy of the present formulation for panel flutter and sonic fatigue is verified and shown in Fig. 2 and Table 1 for panel flutter and sonic fatigue, respectively. The Fokker-Planck-Kolmogorov (FPK) Equation [18] method is an exact solution to the single degree-of-freedom forced Duffing equation. The finite element/equivalent linearization (FE/EL) approach assumes that the equivalent linearizd system obtained after the application of equivalent linearization technique is also stationary Gaussian, while the present time domain 
numerical integration method does not assume that the displacement response is Gaussian, therefore, the present method should be more accurate and realistic.

Table 1. Comparison of RMS of $\left(\mathrm{W}_{\max } / \mathrm{h}\right)$ for a simply supported rectangular $(15 \times 12 \times 0.040 \mathrm{in}$.) isotropic plate using different methods and number of modes

\begin{tabular}{c|c|c|c|c}
\hline SPL & FPK [18] & \multicolumn{2}{|c|}{ FE/EL [19] } & Present \\
$(\mathrm{dB})$ & 1 mode & 1 mode & 4 modes & 4 modes \\
\hline 90 & 0.249 & 0.238 & 0.238 & 0.266 \\
100 & 0.592 & 0.532 & 0.533 & 0.489 \\
110 & 1.187 & 1.030 & 1.031 & 1.092 \\
120 & 2.200 & 1.902 & 1.905 & 2.113 \\
\hline
\end{tabular}

The maximum deflection is located at the three-quarter length from the leading edge. $(3 a / 4, a / 2)$ for the panel flutter, however, it is at the plate center $(a / 2, a / 2)$ for sonic fatigue. For a fixed SPL, the location of the maximum deflection thus moves from the plate center towards the three-quarter length as the dynamic pressure increases. On the other hand, for a fixed dynamic pressure, the location of the maximum deflection moves towards the center from the three-quarter length by increasing the SPL. The maximum deflection for the combined acoustic and aerodynamic loading case, therefore, is not at one fixed location, it can be anywhere between the plate center and three-quarter from the leading edge.

The deflection results shown in Fig. 1 indicate that: (i) the superposition method does not applicable to nonlinear system, (ii) for a given SPL, the RMS deflection at $\lambda=0$ is higher than the deflection at $0<\lambda<\lambda_{\text {cr, }}$, this is due to the increase of panel frequency with increasing of $\lambda$, thus increase the stiffness of the panel, and (iii) the RMS deflection at large dynamic pressure $\left(\lambda \gg>\lambda_{c r}\right)$ are always higher than those at $\lambda=0$. Thus important conclusions can be drawn for design and analysis of surface panels at supersonic speeds: (i) for $\lambda<<\lambda_{\mathrm{cr}}$, only acoustic loading or sonic fatigue has to be considered, and (ii) for $\lambda>\lambda_{\mathrm{cr}}$, both acoustic and aerodynamic loads have to be considered.

Representative panel behavior at five loading combinations are presented in Figs. 3-7. Those five loading combinations correspond to $\mathrm{A}$ to $\mathrm{E}$ shown in Fig. 1. The maximum deflection and maximum strain response time histories, probability distribution and PSD for each loading case are presented. Figures 3 and 4 show the random response at $\mathrm{SPL}=100$ and $120 \mathrm{~dB}$ and $\lambda=0$ (sonic fatigue; points $\mathrm{A}$ and $\mathrm{B}$ in Fig. 1), respectively. At the low $100 \mathrm{~dB}$ SPL, the panel basically experiences a small deflection linear random vibration dominated by the fundamental $(1,1)$ mode. The panel motion at the high $120 \mathrm{~dB}$ SPL, however, is clearly a large deflection nonlinear random vibration. This is demonstrated by the peaks in PSD plots that are broadening and shifting to the higher frequency and by the presence of nonzero-mean inplane strain shown in strain plots.

At $\lambda=800$ and 0 SPL (panel flutter; point C in Fig. 1), the panel. response shown in Fig. 5 is a large amplitude limit cycle motion. The displacement probability density and the PSD of $\left(\mathrm{W}_{\max } / \mathrm{h}\right)$ both describe a periodic motion. The maximum strain time history shows clearly the effect of large inplane strain component due to large amplitude periodic motions.

The panel responses at the combined loads of $\lambda=800$ and SPL $=100$ and $120 \mathrm{~dB}$ (points D and $\mathrm{E}$ in Fig. 1) are shown in Figs. 6 and 7, respectively. The maximum deflection and strain time histories show the nonlinear large deflection vibrations dominated by the fundamental mode and the presence of inplane strain components.

\subsection{Rectangular Composite Plate}

Nonlinear response of composite panels under aerodynamic and acoustic pressures can be determined using the present formulation and solution procedure. As an example, a clamped rectangular graphite-epoxy plate of eight layers [0/45/-45/90]s is analyzed. The dimensions and material properties of the panel are:
$\mathrm{a}=15$ in. $(38.1 \mathrm{~cm})$
$\mathrm{b}=12$ in. $(30.5 \mathrm{~cm})$
$\mathrm{h}=0.048$ in. $(0.122 \mathrm{~cm})$

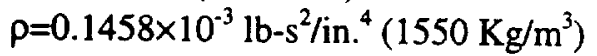
$v_{12}=0.22$

$$
\begin{aligned}
& \mathrm{E}_{1}=22.5 \mathrm{Msi}(155 \mathrm{GPa}) \\
& \mathrm{E}_{2}=1.17 \mathrm{Msi}(8.07 \mathrm{GPa}) \\
& \mathrm{G}_{12}=0.66 \mathrm{Msi}(4.55 \mathrm{GPa}) \\
& \mathrm{G}_{23}=0.44 \mathrm{Msi}(3.03 \mathrm{Gpa})
\end{aligned}
$$


The inplane edges are immovable and the plate is modeled with a $12 \times 12$ mesh. The number of system equations in structure node DOF $\left\{W_{b}\right\}$ is of 363 . The system equations are reduced to the modal coordinates using the lowest $n$ modes in increasing frequency order. The RMS $W_{\max } / \mathrm{h}$ at $120 \mathrm{~dB}$ SPL and $\lambda=800$ using different number of modes are shown in Table 2 . The results show that a 20 - or 25 -mode model would yield a converged RMS maximum deflection.

To demonstrate the advantage of using modal participation defined in Eq. (9), the participation values for the 25 -mode model are shown in Table 3. By retaining those 13 modes with participation value $>1 \%$ in the analysis, the RMS $\mathrm{W}_{\max } / \mathrm{h}$ is 0.8124 at $120 \mathrm{~dB}$ SPL and $\lambda=800$. Using the 13 most contributing modes, the RMS $\left(\mathrm{W}_{\max } / \mathrm{h}\right)$ versus nondimensional dynamic pressure $\lambda$ at SPL of $0,100,110$ and $120 \mathrm{~dB}$ are shown in Fig. 8. Similar conclusion from the isotropic panel can be drawn for the composite panel, that is, at low dynamic pressure $\left(\lambda<<\lambda_{\text {cr }}\right)$ only acoustic loading needs to be considered, and at high dynamic pressure $\left(\lambda>\lambda_{\mathrm{cr}}\right)$ both aerodynamic and acoustic loads have to be considered for the design and analysis of surface panels at supersonic flow. Response time history, probability distribution and PSD are not repeated for the composite panel.

Table 2. RMS ( $\left.W_{\max } / \mathbf{h}\right)$ for a clamped rectangular graphite-epoxy panel at $120 \mathrm{~dB}$ SPL and $\lambda=800$ using different number of modes

\begin{tabular}{cc}
\hline Number of modes, $\mathrm{n}$ & $\mathrm{RMS}\left(\mathrm{W}_{\max } / \mathrm{h}\right)$ \\
\hline 1 & 0.5557 \\
2 & 0.5845 \\
6 & 0.7814 \\
9 & 0.7798 \\
16 & 0.8279 \\
20 & 0.8110 \\
25 & 0.8183 \\
\hline Selected 13 modes & 0.8124 \\
\hline
\end{tabular}

Table 3. Modal participation values for a clamped rectangular graphite-epoxy panel at $120 \mathrm{~dB}$ SPL and $\lambda=800$ using the lowest 25 modes

\begin{tabular}{ccccccccccccc}
\hline Mode number & 1 & 2 & 3 & 4 & 5 & 6 & 7 & 8 & 9 & 10 & 11 & 12 \\
Participation, \% & 36.72 & 5.24 & 19.30 & 4.25 & 4.01 & 7.67 & 1.71 & 0.76 & 0.33 & 1.54 & 4.77 & 0.35 \\
\hline 13 & 14 & 15 & 16 & 17 & 18 & 19 & 20 & 21 & 22 & 23 & 24 & 25 \\
0.28 & 4.19 & 0.38 & 0.99 & 1.38 & 2.55 & 0.21 & 0.27 & 0.53 & 0.33 & 0.54 & 0.14 & 1.54 \\
\hline
\end{tabular}

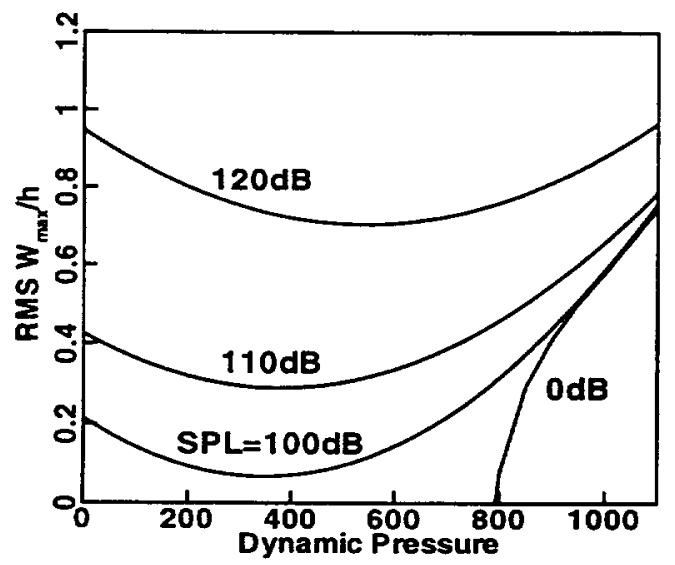

Fig. 8 RMS maximum deflection of a clamped rectangular graphite-epoxy plate 


\section{CONCLUDING REMARKS}

A finite element time domain modal formulation is presented for the analysis of nonlinear response of composite panels subjected to combined acoustic and aerodynamic pressures. The advantage of using modal participation for retaining the most contributing modes was demonstrated. For panels at supersonic flow, only acoustic excitations (sonic fatigue) are to be considered for $\lambda<<\lambda_{c r}$, and both acoustic and aerodynamic pressures have to be considered for $\lambda>\lambda \mathrm{cr}$. Future extension of the present work includes the combined acoustic, aerodynamic and thermal loads. Arbitrary flow directions and curved panels will also be considered.

\section{ACKNOWLEDGMENTS}

The first and the second authors would like to acknowledge the partial support by grant F33615-91-C-3205, AFRL. The third author would like to acknowledge the support by grant NAG1-2150, NASA Langley Research Center and by grant F33615-91-C-3205, AFRL.

\section{REFERENCES}

1. Vaicaitis, R., "Recent Advances of Time Domain Approach for Nonlinear Response and Sonic Fatigue," Proceedings of $4^{\text {th }}$ International Conference on Structural Dynamics: Recent Advances, Institute of Sound and Vibration Research, University of Southampton, UK, July 1991, pp. 84-103

2. Vaicaitis, R., "Time Domain Approach for Nonlinear Response and Sonic Fatigue of NASP Thermal Protection Systems," Proceedings of $32^{\text {nd }}$ Structures, Structural Dynamics and Materials Conference, Baltimore, MD, April 1991, pp. 2685-2708.

3. Clarkson, B. L., "Review of Sonic Fatigue Technology," NASA CR-4587, 1994.

4. Wolfe, H. F., Shroyer, C. A., Brown, D. L. and Simmons, L. W., "An Experimental Investigation of Nonlinear Behavior of Beams and Plates Excited to High Levels of Dynamic Response," WL-TR-96-3057, WrightPatterson AFB, OH, 1995.

5. Rudder, F. F. and Plumblee, H. E., "Sonic Fatigue Design Guide for Military Aircraft," AFFDL-TR-74-112, Wright-Patterson AFB, OH, 1975.

6. Holehouse, I., "Sonic Fatigue Design Guide Techniques for Advanced Composite Airplane Structures," AFWAL-TR-80-3019, Wright-Patterson AFB, OH, 1980.

7. Amold, R. R. and Vaicaitis, R., "Nonlinear Response and Fatigue of Surface Panels by the Time Domain Monte Carlo Approach," WRDC-TR-90-3081, Wright-Patterson AFB, OH, 1992.

8. Vaicaitis, R. and Kavallieratos, P. A., "Nonlinear Response of Composite Panels to Random Excitation," Proceedings of $34^{\text {th }}$ Structures, Structural Dynamics and Materials Conference, La Jolla, CA, April 1993, pp. 1041-1049.

9. Dowell, E. H., "Panel Flutter: A Review of the Aeroelastic Stability of Plates and Shells," AIAA Journal, Vol. 8, No. 3, 1970, pp. 385-399.

10. Bismarck-Nasr, M. N., "Finite Element Analysis of Aeroelasticity of Plates and Shells," Applied Mechanics Review, Vol. 45, No. 12, 1992, pp.461-482.

11. Mei, C., Abdel-Motagaly, K. and Chen, R., "A Review of Nonlinear Panel Flutter at Supersonic and Hypersonic Speeds," Proceeding of CEAS/AIAA/ICASE/NASA Langley International Forum on Aeroelasticity and Structural Dynamics, Williamsburg, VA, June 1999.

12. Laurenson, R. M. and McPherson, J. I., "Design Procedures for Flutter-Free Surface Panels," NASA CR-2801, 1977.

13. Zhou, R. C., Xue. D. Y. and Mei. C., "Finite Element Time Domain Modal Formulation for Nonlinear Flutter of Composite Panels," AlAA Journal, Vol. 32, No. 10, 1994, pp.2044-2052.

14. Mei, C. and Chen, R. R., 'Finite Element Nonlinear Random Response of Composite Panels of Arbitrary Shape to Acoustic and Thermal Loads Applied Simultaneously," WL-TR-97-3085, Wright-Patterson AFB, OH, 1997.

15. Robinson, J. H., "Finite Element Formulation and Numerical Simulation of the Large Deflection Random Vibration of Laminated Composite plates," MS Thesis, Old Dominion University, Norfolk, VA, 1990. 
16. Green, P. D., and Killey, A., "Time Domain Dynamic Finite Element Modeling in Acoustic Fatigue Design," Proceedings of $6^{\text {th }}$ International Conference on Structural Dynamics: Recent Advances, Institute of Sound and Vibration Research, University of Southampton, UK, 1997, pp.1007-1026.

17. Tessler, A. and Hughes, T. J. R., "A Three-node Mindlin Plate Element with Improved Transverse Shear," Computer Methods in Applied Mechanics and Engineering, Vol. 50, 1985, pp. 71-91.

18. Bolotin, V. V., "Random Vibration of Elastic Systems," Martinus Nijhoff Publishers, 1984, pp. 290-292, 314316.

19. Chiang, C. K., "A Finite Element large Deflection Multiple-Mode Random Response Analysis of Complex panel with Initial Stresses Subjected to Acoustic Loading," Ph. D. Dissertation, Old Dominion University, Norfolk, VA, May 1983.
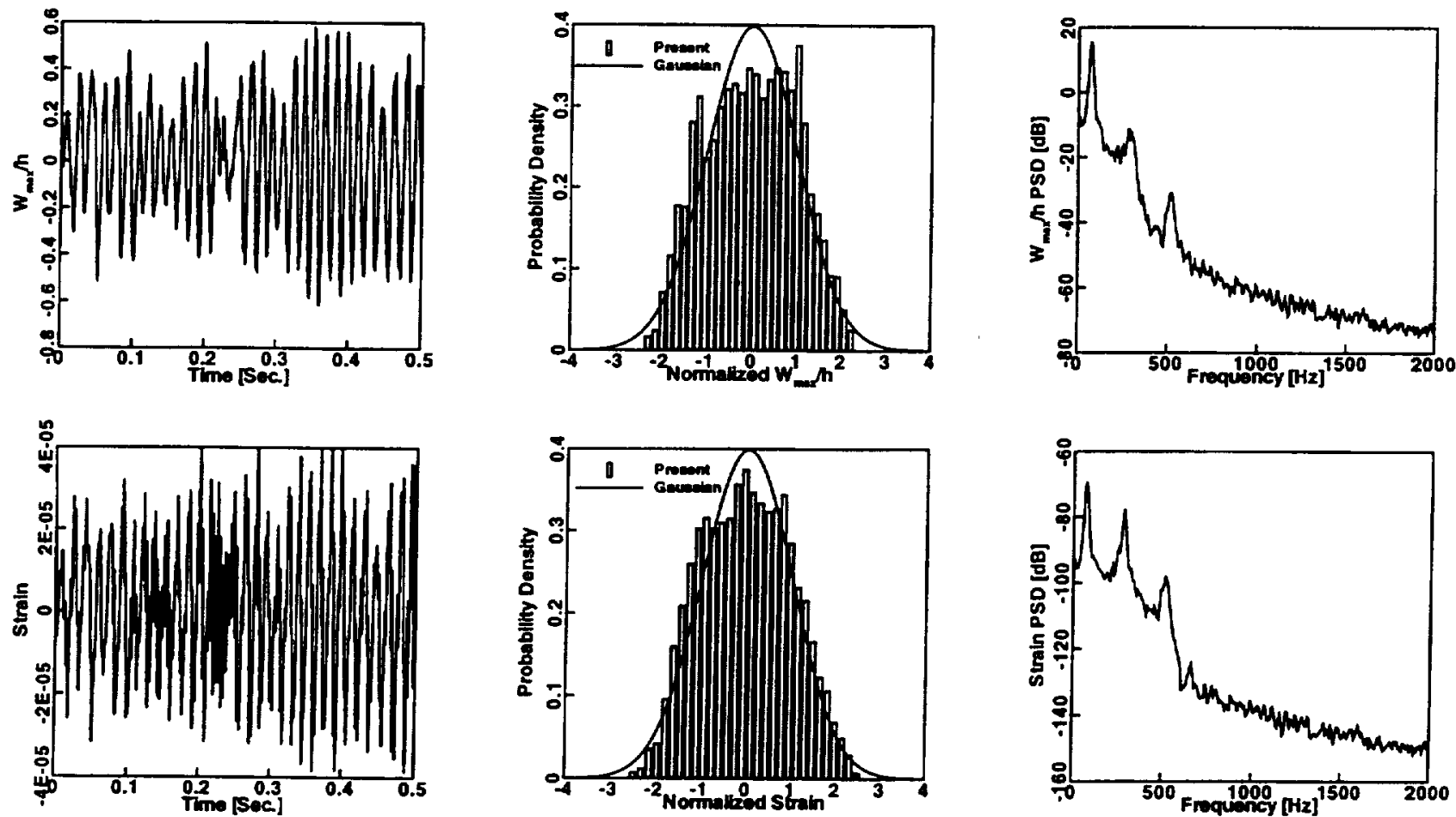

Fig. 3 Random Response of a simply supported square isotropic plate at $100 \mathrm{~dB}$ SPL and $\lambda=0$ 

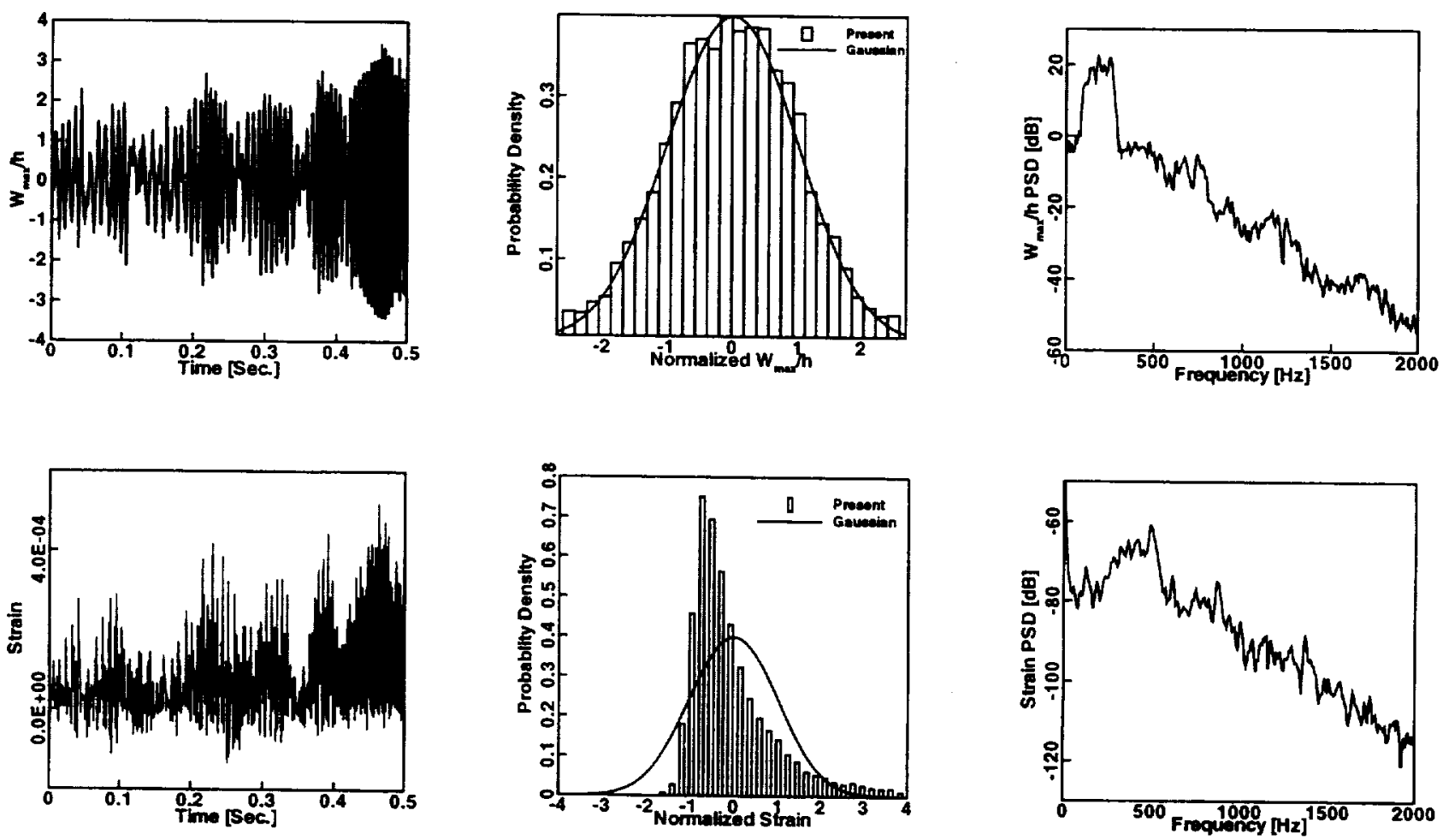

Fig. 4 Random Response of a simply supported square isotropic plate at $120 \mathrm{~dB}$ SPL and $\lambda=0$
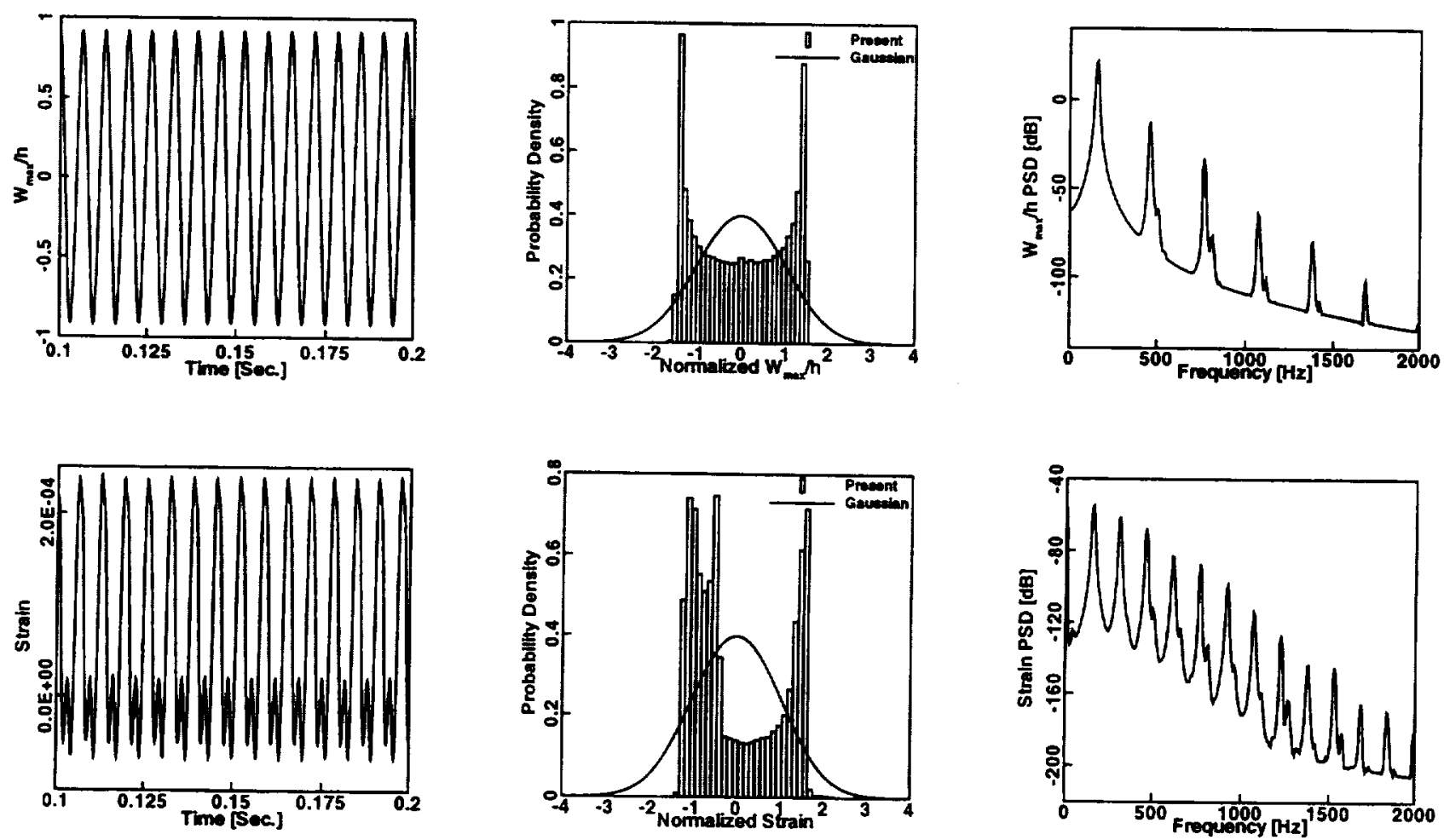

Fig. 5 Response of a simply supported square isotropic plate at $0 \mathrm{~dB}$ SPL and $\lambda=800$ 

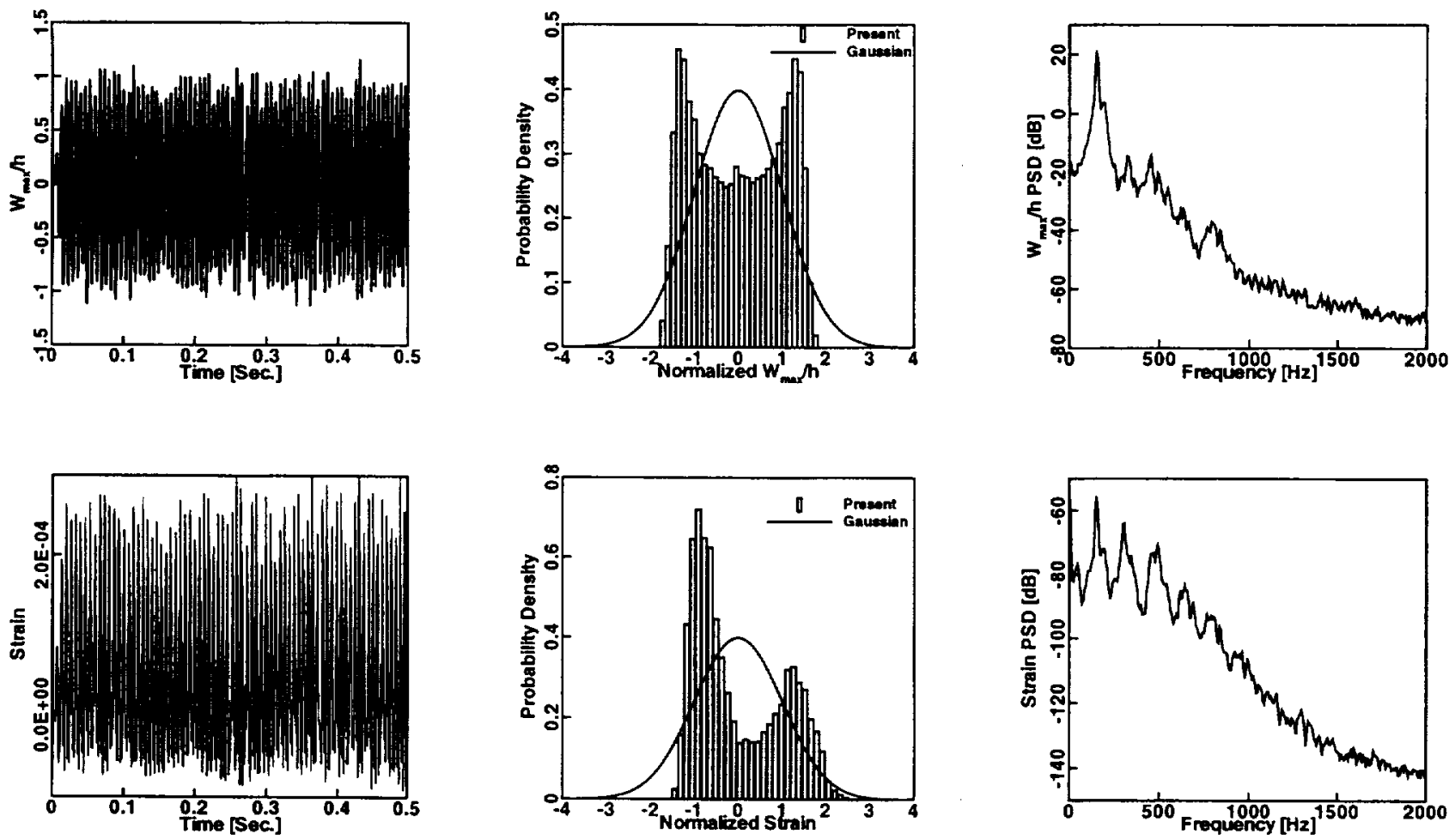

Fig. 6 Random Response of a simply supported square isotropic plate at $100 \mathrm{~dB}$ SPL and $\lambda=800$
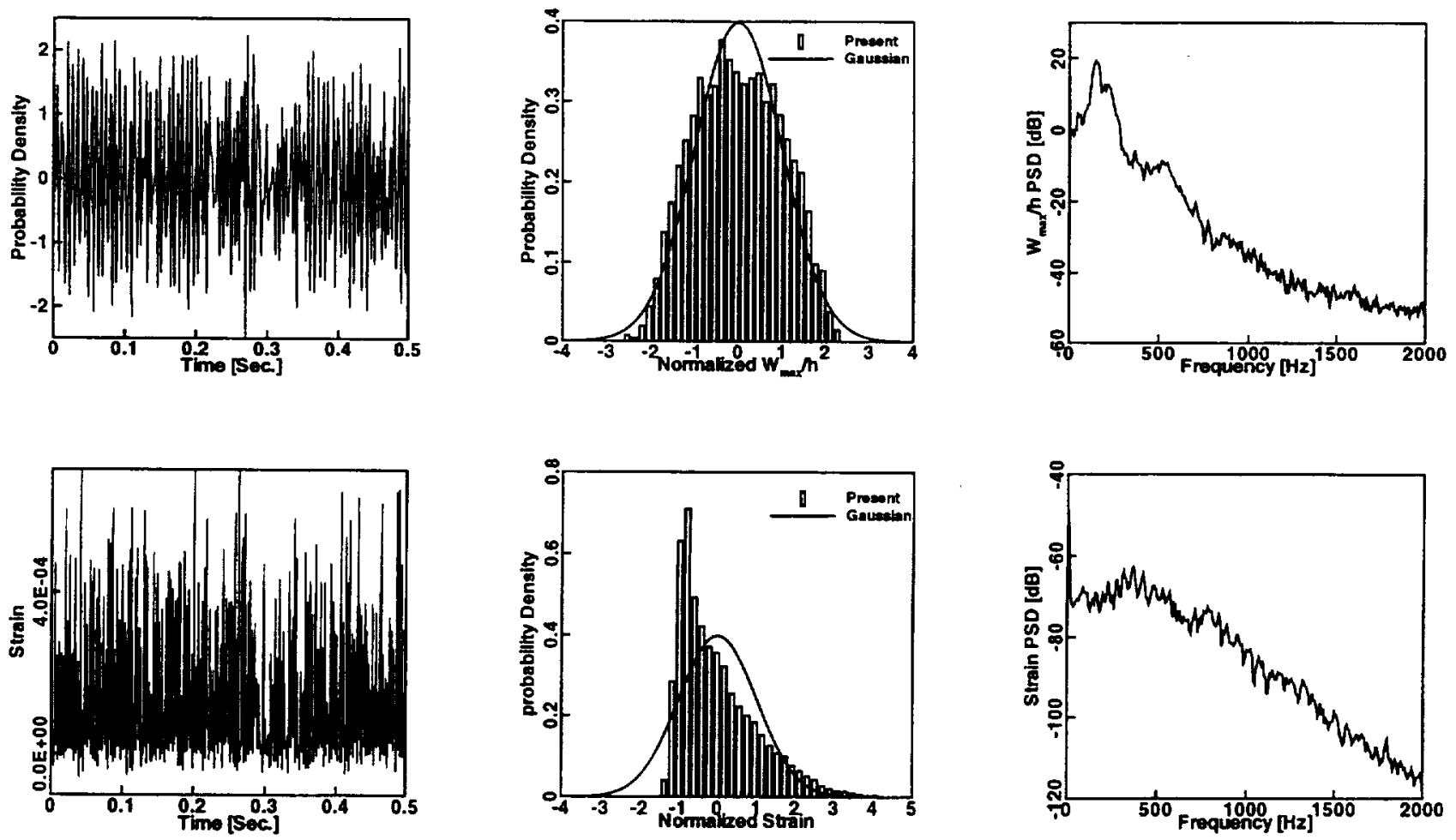

Fig. 7 Random Response of a simply supported square isotropic plate at $120 \mathrm{~dB}$ SPL and $\lambda=800$ 
